\title{
Accurate measurements of the thermal diffusivity of thin films and thin filaments using lock-in thermography
}

\author{
by A. Salazar*, A. Mendioroz*, R. Fuente* and R. Celorrio** \\ *Departamento de Física Aplicada I, Escuela Técnica Superior de Ingeniería, Universidad del País Vasco, Alameda \\ Urquijo s/n, 48013 Bilbao, Spain, agustin.salazar@ehu.es \\ **Departamento de Matemática Aplicada, EUITIZ, Universidad de Zaragoza, Campus Río Ebro, Edificio Torres \\ Quevedo, 50018 Zaragoza, Spain, celorrio@unizar.es
}

\begin{abstract}
In lock-in thermography the thermal diffusivity can be obtained from the slope of the linear relation between the phase of the surface temperature and the distance to the heating spot. However, in the case of thin samples, the linearity is not found. In this work we identify heat conduction to the surrounding gas as the mechanism responsible for the lost of linearity. Accordingly accurate measurements of the thermal diffusivity require thin samples to be kept in vacuum. Measurements performed in a wide variety of films and filaments confirm the validity of the method.
\end{abstract}

\section{Introduction}

Lock-in thermography consists in illuminating the sample by an intensity modulated light beam and detecting the oscillating component of the temperature rise by means of an infrared video camera connected to a lock-in module. Lock-in thermography has been broadly used to measure the thermal diffusivity $(D)$ of a wide variety of materials. In a typical setup, a modulated laser beam is focused onto the sample surface while the infrared camera records the surface temperature. Under ideal conditions (absence of heat losses and absence of diffraction effects), for a given modulation frequency $(f)$, both the natural logarithm of the amplitude of the temperature, $\operatorname{Ln}(T)$, and its phase $(\Psi)$ depend linearly on the distance to the heating spot, with the same slope given by $m=-(\pi f / D)^{0.5}$, from which the thermal diffusivity can be obtained (the so-called slope method). This simple method has been successfully applied to good thermal conductors, but it fails when dealing with poor thermal conductors.

In this work we develop a complete theoretical model to calculate the surface temperature of thin films and thin filaments, by including the effect of heat losses. In this way, conduction to the surrounding gas has been identified as the most disturbing effect preventing from the direct use of the slope method to measure the thermal diffusivity. Consequently, by keeping the sample in vacuum the slope method can be used to obtain the accurate $D$ value. Measurements performed in a wide variety of thin films and thin filaments confirm the validity of the conclusion.

\section{Theory}

We have calculated the oscillating temperature of an opaque thin slab of thickness $L$ and of a thin filament of radius a, illuminated by a modulated $(\omega=2 \pi f)$ and tightly focused laser beam is calculated $[1,2]$. Heat losses by conduction and convection to the surrounding gas, as well as by radiation, have been taken into account. Simple expressions have been found for the following cases.

In the absence of heat losses the surface temperature for a thin slab and for a thin filament write respectively

$$
T(r) \approx \frac{P}{4 \pi K L} K_{o}(q r) \quad \text { and } \quad T(z) \approx \frac{P}{2 \pi K q a} e^{-q|z|}
$$

where $P$ is the laser beam power, $K$ is thermal conductivity, $q=(i \omega / D)^{0.5}$ is the thermal wave vector and $K_{0}$ is the Kelvin function of zeroth order. According to these expressions the natural logarithm of the amplitude of the temperature, $\operatorname{Ln}(T)$, and its phase $(\Psi)$ depend linearly on the distance to the heating spot, with the same slope given by $m_{\operatorname{Ln}(T)}=m_{\Psi}=-(\pi f / D)^{0.5}$, from which the thermal diffusivity can be obtained.

If only heat losses by convection and radiation are considered (i.e. neglecting heat conduction to the gas), Eqs. (1) are still valid for the surface temperature of a thin slab and of a thin filament, but changing the thermal wave vector $q$ by $q^{\prime}$, which writes: $q^{\prime 2}=q^{2}+2 h / K L$ for a thin film and $q^{\prime 2}=q^{2}+2 h / K a$ for a thin filament. Here $h$ is the combined heat transfer coefficient of convection and radiation. As before, $\operatorname{Ln}(T)$ and $\Psi$ depend linearly on the distance to the heating spot, but now 
the slope of the phase is larger than the slope of the amplitude. However, the product of both slopes verifies $m_{\mathrm{Ln}(T)} \times m_{\Psi}=-$ $\pi f / D$, which allows to retrieve the thermal diffusivity.

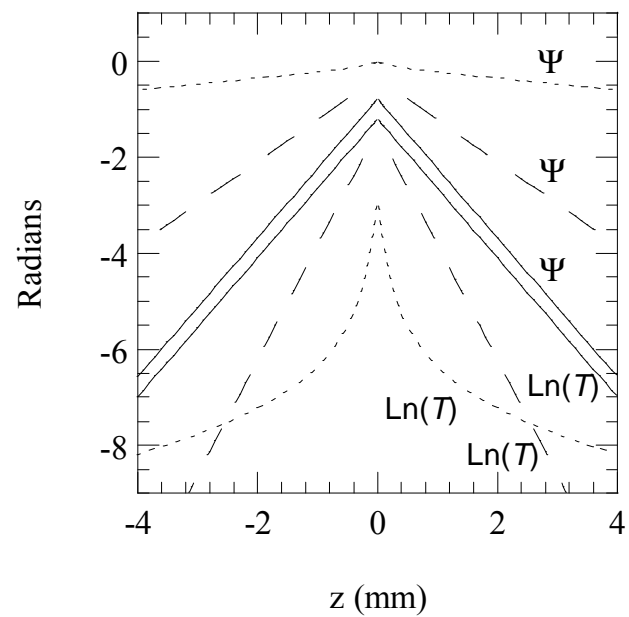

Fig. 1. Simulations of the lateral dependence of $\operatorname{Ln}(T)$ and $\psi$ of a polymeric filament of $60 \mu \mathrm{m}$ of diameter. Continuous lines: Absence of heat losses. Dashed lines: Heat losses by convection and radiation. Dotted lines: All heat losses. $f=0.1 \mathrm{~Hz}$.

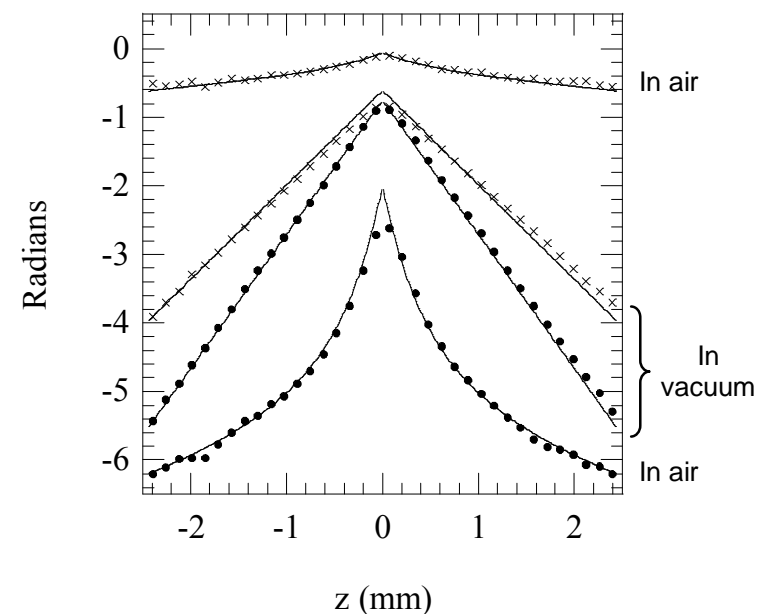

Fig. 2. Experimental values of $L n(T)$ (dots) and $\psi$ (crosses) of the surface temperature of a human hair of $60 \mu \mathrm{m}$ of diameter measured at $f=0.12 \mathrm{~Hz}$. Measurements were performed in air and in vacuum. The solid lines are the best fittings with $D_{\text {hair }}=0.14 \mathrm{~mm}^{2} / \mathrm{s}$.

However, when the three heat loss mechanisms are present the linearity is lost. It is worth noting that this lost of linearity is more pronounced as the thermal diffusivity of the sample as well as its size (thickness in films, radius in filaments) decrease. This behavior is illustrated in figure 1, where the lateral dependence of $\operatorname{Ln}(T)$ and $\Psi$ is calculated for a polymeric filament of $60 \mu \mathrm{m}$ in diameter. As can be seen, the linearity is lost when the heat conduction to the gas is included. This means that this last one is the most disturbing mechanism preventing from the direct use of the slope method to measure the thermal diffusivity of thin films and thin filaments. However, by keeping the sample in vacuum the slope method can be used to measure the thermal diffusivity accurately.

\section{Experimental results}

In order to verify the last conclusion we have used a lock-in thermography setup to measure the temperature profile of thin films and thin filaments heated by a modulated and focused (spot about $50 \mu \mathrm{m}$ ) laser beam. Samples with very different diffusivity values (from polymers to metals) and sizes (filaments between 7 and $125 \mu \mathrm{m}$, and free standing films from 10 to $250 \mu \mathrm{m}$ ) have been studied. In all cases when the sample is in a vacuum chamber the accurate thermal diffusivity is obtained. As an example, we show in figure 2 the results obtained on a human hair at low frequency. As can be seen, measurements performed in air (symbols) show the predicted lost of linearity in agreement with the theoretical predictions (continuous lines). However, when the hair is maintained in vacuum the linearity is recovered, since radiation is the only acting mechanism of heat loss. Therefore the thermal diffusivity can be obtained from the product of both slopes: $D_{\text {hair }}=$ $0.14 \pm 0.01 \mathrm{~mm}^{2} / \mathrm{s}$. This value is typical of biological samples.

This work has been supported by the Ministerio de Educación y Ciencia (MAT2008-01454).

\section{REFERENCES}

[1] Mendioroz A., Fuente-Dacal R., Apiñaniz E. and Salazar A., Rev. Sci. Instrum. 80, 074904-074912 (2009).

[2] Salazar A., Mendioroz A., Fuente R., Appl. Phys. Lett. 95, 121905-121907 (2009). 